\title{
Universiteit
}

Leiden

The Netherlands

\section{Scaling Unidimensional Models with Multiple Correspondence Analysis}

Warrens, M.J.; Heiser, W.J.; Greenacre M., Blasius J.

\section{Citation}

Warrens, M. J., \& Heiser, W. J. (2006). Scaling

Unidimensional Models with Multiple Correspondence

Analysis. In B. J. Greenacre M. (Ed.), Multiple

Correspondence Analysis and Related Methods (pp.

219-235). London: Chapman \& Hall/CRC. Retrieved from https://hdl.handle.net/1887/14576

Version:

License:

Downloaded from: $\quad$ https://hdl.handle.net/1887/14576

Note: To cite this publication please use the final published version (if applicable). 


\section{Scaling Unidimensional Models with Multiple Correspondence Analysis}

\section{Matthijs J. Warrens and Willem J. Heiser}

\section{CONTENTS}

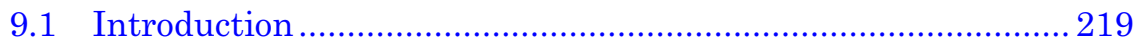

9.2 The dichotomous Guttman scale ....................................... 221

9.3 The Rasch model................................................................... 224

9.4 The polytomous Guttman scale ........................................... 228

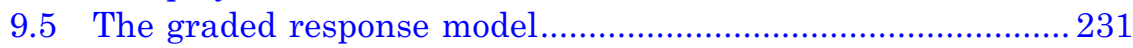

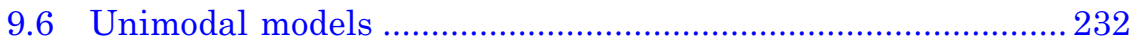

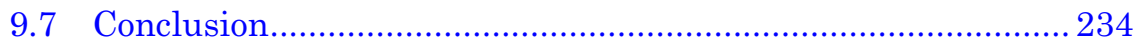

\subsection{Introduction}

This chapter discusses the application of multiple correspondence analysis (MCA) as a method of scaling. For this type of application of MCA, several well-known unidimensional models from the psychometric literature will be considered. All of these models are characterized by item and person parameters. The objective of this chapter is to determine what information on these parameters can be obtained from applying MCA when the unidimensional models are used as gauges. "Gauge" is a term introduced by Gifi (1990) to denote benchmark data sets, or mechanisms to generate such data sets, for studying the behavior of general-purpose data analysis methods, such as MCA and simple correspondence analysis (CA). Some of the basics on this topic are reviewed, but many new insights and results are also presented. 
The models discussed in this chapter can be classified by three different aspects, i.e., each model is either

Deterministic or probabilistic

Dichotomous or polytomous

Monotonic or unimodal

It follows that there are $2 \times 2 \times 2=8$ possible categories to classify a model, but only six of them will actually be discussed. For the categories corresponding to the probabilistic and unimodal options, no successful applications of MCA are currently available. Only for the two deterministic unimodal models are there some ideas for scaling with MCA (see Section 9.6). For each of the four deterministic categories, only one candidate model seems available, while for the probabilistic categories there are numerous possible models that can be selected. However, for three of the deterministic models, an additional distinction is presented between different possible forms of the models. This distinction provides several new insights into the question of when and especially how to apply MCA.

With MCA, CA, or related methods, the structure of the multivariate data is often visualized in a two-dimensional representation. A common conviction is that when one applies a technique such as MCA to data satisfying a unidimensional model, the resulting two-dimensional representation will reflect some sort of horseshoe (see van Rijckevorsel 1987; Gifi 1990; Hill and Gauch 1980). More precisely, the phenomenon will be called an arch if the graph reflects a quadratic function. If, in addition, the ends of this arch bend inward, the phenomenon will be called a horseshoe. It will be shown that unidimensionality is not a sufficient condition for a horseshoe, that is, the data may be unidimensional in terms of a model, but a method such as MCA will not produce a horseshoe in two or any other dimensions. Furthermore, it will be shown that, with an appropriate analysis, most relevant information in terms of item and person parameters can be found in the first MCA dimension.

We end this section with some words on notation. The quantification of category $j\left(1, \ldots, J_{q}\right)$ of item $q(1, \ldots, Q)$ on dimension $s(1, \ldots, S)$ is denoted by $y_{q s}^{j}$. In our context, $J_{q}$ is the same for all items, and we use the notation $J$ for this constant number of categories per item. (This is different from standard MCA notation, where $J$ is the total number of $y$ categories for all items.) The vector $\mathbf{y}_{s}^{j}$ then denotes the quantifications of the jth category for all items on dimension $s$, and $\mathbf{y}_{s}$ denotes all quantifications on dimension $s$. Furthermore, let $\mathbf{x}_{s}$ denote the 


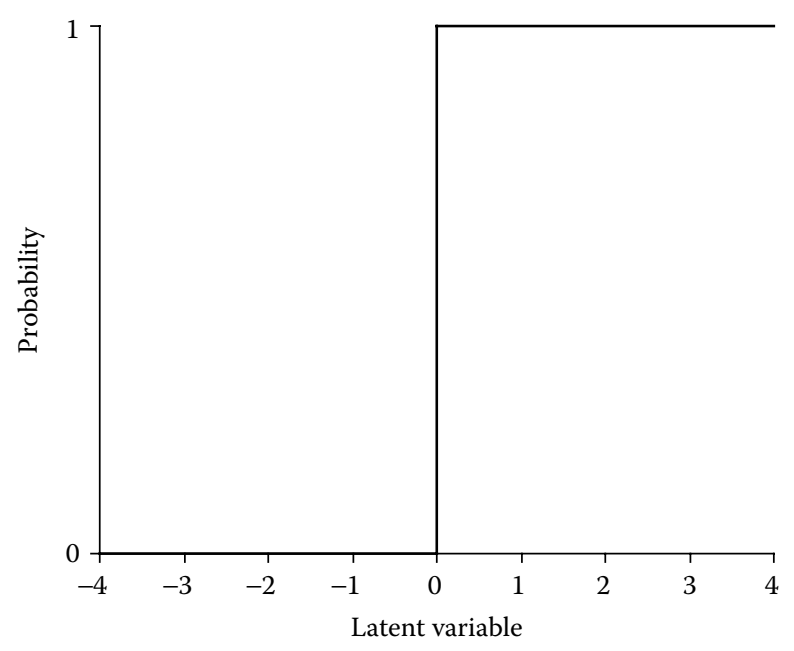

Figure 9.1 Item function of the DGS.

person scores on dimension $s$. Let $u$ denote a latent variable, and $\delta_{q}$ a location parameter of item $q$. Explicit formulas for the item functions that relate $u$ to $\delta_{q}$ will not be given; only their shapes will be shown in the figures.

\subsection{The dichotomous Guttman scale}

The simplest and oldest model considered in this chapter is the dichotomous Guttman scale (DGS), named after the person who popularized the model with the method of scalogram analysis. With the DGS, each item is characterized by a step function, as is shown in Figure 9.1.

Guttman (1950b, 1954) advocated the practical properties of the DGS, but earlier, other authors (for example, Walker 1931) also noted the special structure of the data matrix and the possibilities of ordering both persons and items. Parameters of the DGS are only unique in terms of their order, that is, they form an ordinal scale. Often-used estimates are the sum score as an index for persons and the proportion correct for items.

Both the DGS and the application of MCA to the DGS were thoroughly studied by Guttman (1950b, 1954). Guttman (1950b) derived that all relevant information for the ordinal properties of the DGS is contained in $\mathbf{y}_{1}$ for item categories and $\mathbf{x}_{1}$ for persons. Guttman 
(1950b, 1954) also studied the quantifications and scores for dimensions $s>1$. With $Q$ items there are $Q+1$ possible score patterns in a DGS. The DGS can be referred to as complete if all possible score pattern are present and uniform if all present score patterns occur equally often. The matrix $\mathbf{M}$ below contains an example of a complete and uniform DGS of three items.

$$
\mathbf{M}=\left[\begin{array}{lll}
1 & 1 & 1 \\
2 & 1 & 1 \\
2 & 2 & 1 \\
2 & 2 & 2
\end{array}\right] \Rightarrow \mathbf{Z}=\left[\begin{array}{llllll}
1 & 0 & 1 & 0 & 1 & 0 \\
0 & 1 & 1 & 0 & 1 & 0 \\
0 & 1 & 0 & 1 & 1 & 0 \\
0 & 1 & 0 & 1 & 0 & 1
\end{array}\right] \rightarrow\left[\begin{array}{llllll}
1 & 1 & 1 & 0 & 0 & 0 \\
0 & 1 & 1 & 1 & 0 & 0 \\
0 & 0 & 1 & 1 & 1 & 0 \\
0 & 0 & 0 & 1 & 1 & 1
\end{array}\right]
$$

The matrix $\mathbf{Z}$ is the indicator matrix resulting from $\mathbf{M}$, and the right matrix shows $\mathbf{Z}$ with the columns permuted such that the elements of $\mathbf{y}_{1}^{1}$ and $\mathbf{y}_{1}^{2}$ come together and are ordered. The data matrix $\mathbf{M}$ reflects a scalogram structure, whereas the (permuted) indicator matrix $\mathbf{Z}$ reflects a parallelogram structure.

For the DGS, Guttman (1950b) showed that

The ordering of the proportion correct is reflected in both $\mathbf{y}_{1}^{1}$ and $\mathbf{y}_{1}^{2}$

The ordering of the sum score is reflected in the person scores $\mathbf{x}_{1}$ $\mathbf{x}_{2}$ has a quadratic relation to $\mathbf{x}_{1}$

Guttman (1950b, 1954) considered even higher polynomial relations between $\mathbf{x}_{1}$ and $\mathbf{x}_{s}$ for $s>2$, but these are outside the scope of this chapter. Note that $\mathbf{y}_{1}$ and $\mathbf{y}_{2}$ do not have a precise quadratic relation, although the relation will have resemblance to a horseshoe or an arch (see Figure 9.2). In fact there are two superimposed arches, one for each category, a result proven by Schriever (1985).

The above result shows that applying MCA to the DGS provides category quantifications that reflect the ordering of items and scores that reflect the ordering of persons. The same result would be obtained by using the proportion item correct and the sum score for indices, respectively. However, all these indices give an ordering for items and persons separately, that is, the indices for items and persons do not imply a simultaneous ordering. However, for a special case of the DGS, a stronger result can be obtained.

For the complete and uniform DGS, Guttman showed that the person scores are proportional to the sum scores. If there are $Q \delta_{q}$ 's 

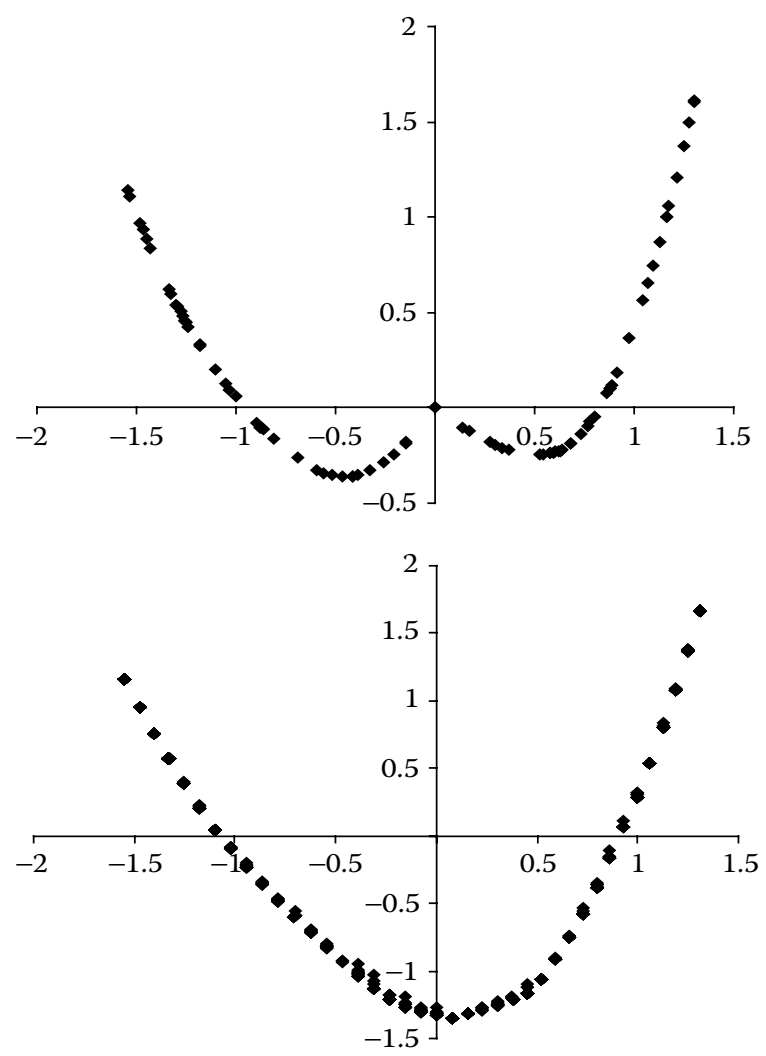

Figure 9.2 First two axes of MCA of DGS for $Q=40, J=2$; category quantifications (above) and person scores (below).

representing location parameters, there are $Q+1 u$ 's indicating the possible person scores. For each pair $u_{q}$ and $u_{q+1}$, a $\delta_{q}$ is required that satisfies $u_{q} \leq \delta_{q} \leq u_{q+1}$. A desirable value for this location parameter $\delta_{q}$ would be

$$
\hat{\delta}_{q}=\frac{u_{q}+u_{q+1}}{2}
$$

The following proposition shows how this estimate for $\delta_{q}$ can be obtained from $y_{q}^{1}$ and $\mathrm{y}_{q}^{2}$, the MCA quantifications of the categories of item $q$ on the first dimension. 
Proposition. Under the condition of a complete uniform DGS, the estimate in Equation 9.1 can be obtained by taking $\delta_{q}=y_{q}^{1}+y_{q}^{2}$.

Proof. With a uniform DGS, each score pattern occurs $T$ times. Without loss of generality we can take $T=1$. Because with a complete uniform scale the $u$ 's are proportional to the sum scores (Guttman 1950b), they are, for convenience, not put in standard scores, but are expressed as real integers, that is, $u_{q}=q$, for $q=1, \ldots, Q+1$, minus the grand mean. For a set of $Q+1$ score patterns, the grand mean value is then given by $1 / 2(Q+2)$. The category quantifications of the item that discriminates between $u_{q}$ and $u_{q+1}$ can be expressed as

$$
\begin{aligned}
& y_{q}^{1}=1 / 2(q+1) ; \\
& y_{q}^{2}=1 / 2(q+1)+1 / 2(Q+1)
\end{aligned}
$$

before the grand mean is subtracted. Centering around the grand mean gives

$$
\begin{aligned}
u_{q} & =q-1 /{ }_{2}(Q+2) \\
y_{q}^{1} & =1 / 2(q+1)-1 / 2(Q+2) \\
& =1 /{ }_{2} q-1 / 2 Q-1 / 2 \\
y_{q}^{2} & =1 / 2(q+1)+1 / 2(Q+1)-1 / 2(Q+2) \\
& =1 / 2 q
\end{aligned}
$$

Hence,

$$
\begin{aligned}
y_{q}^{2}+y_{q}^{1} & =q-1 / 2 Q-1 / 2 \\
& =\delta_{q}
\end{aligned}
$$

\subsection{The Rasch model}

A probabilistic generalization of the DGS is the model proposed by Rasch (1960). In the Rasch model, an item is characterized by a logistic function instead of a step function. Similar to the DGS, the Rasch model is a location family, or a holomorphic model, meaning that the item functions have the same shape but are translations of each other (see Figure 9.3). 


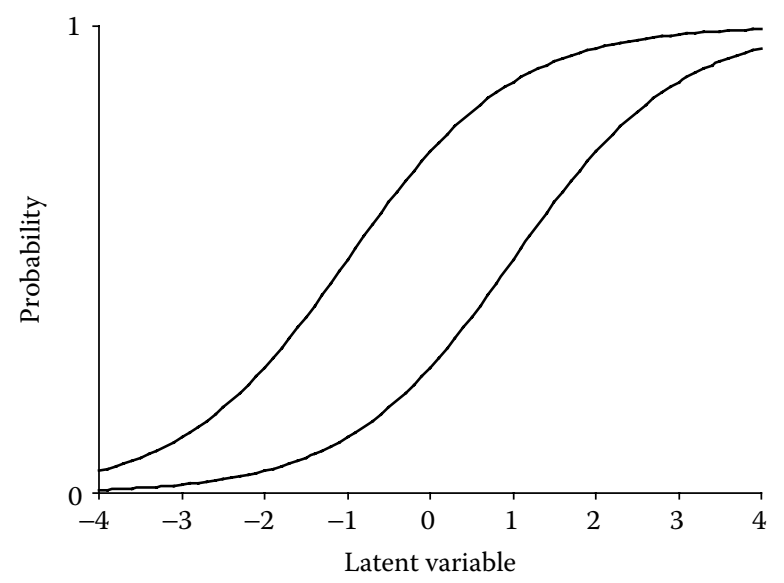

Figure 9.3 Two item functions of the Rasch model.

With a probabilistic dichotomous model, there are $2^{Q}$ possible score patterns for $Q$ items. However, depending on how the Rasch model is specified, some score patterns are more likely to occur than others. For example, if one specifies the Rasch model such that it has relatively steep slopes, then the score patterns will have close resemblance to the score patterns of the DGS. On the other hand, if the Rasch model is specified such that the slopes are not steep, the score patterns will look close to random. The existence of multiple forms of the Rasch model has important consequences for the application of MCA and related methods. A Rasch model with steep slopes will provide a twodimensional solution that is similar to the arch for the DGS. For a Rasch model with shallow slopes, the two-dimensional solution will look like random data without structure (see Figure 9.4).

So, MCA has a limited usefulness in detecting probabilistic unidimensional models. However, instead of looking at both $\mathbf{y}_{1}$ and $\mathbf{y}_{2}$ or $\mathbf{x}_{1}$ and $\mathbf{x}_{2}$, several results indicate that most relevant information for the Rasch models is obtained in $\mathbf{y}_{1}$ and $\mathbf{x}_{1}$.

Schriever (1985) showed that, if the item functions of a dichotomous model are monotonic and have monotone likelihood ratio (Lehmann 1966), then the ordering is reflected in both $\mathbf{y}_{1}^{1}$ and $\mathbf{y}_{1}^{2}$. The item functions of the Rasch model satisfy this more general property. Furthermore, because the category quantifications reflect the ordering of the items, the reciprocal averages, that is, the person scores, will reflect a reasonable ordering of the persons. These ordering properties are 


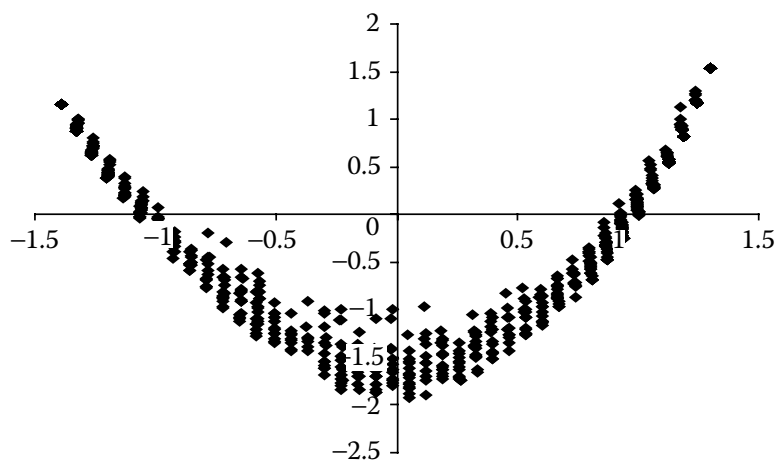

(a)
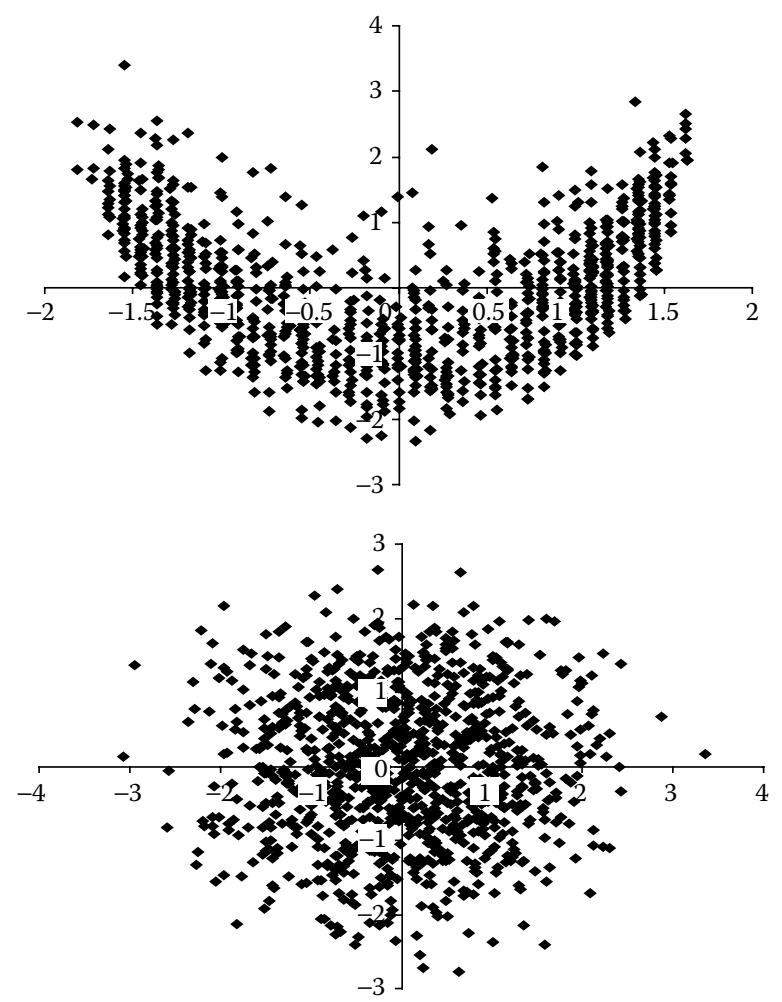

(b)

Figure 9.4 MCA person scores of three Rasch data sets, with $Q=40$ and the same location parameters but decreasing slopes. 

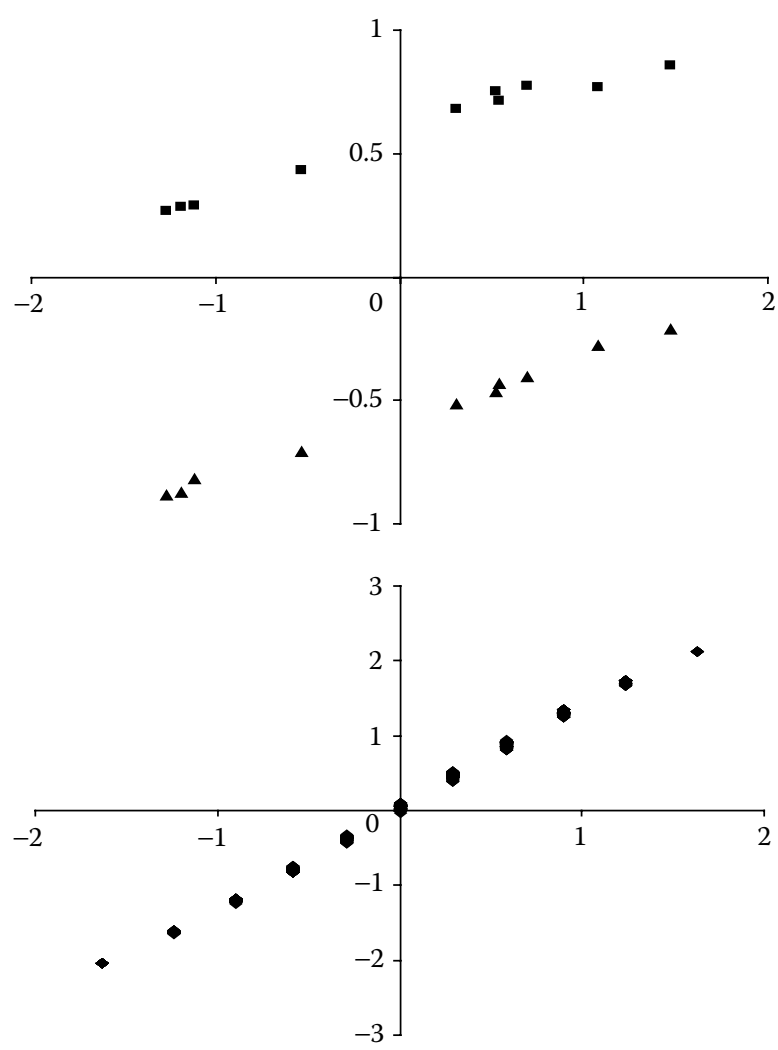

Figure 9.5 The first plot shows the MCA quantifications (vertical) of the two sets of categories plotted vs. item-response-theory estimates for the location parameters (horizontal) of a Rasch data set with $Q=10$. Both sets of quantifications reflect the ordering of the location parameters. The second plot shows the MCA person scores (vertical) vs. the item-response-theory person estimates (horizontal).

visualized in Figure 9.5, where the MCA parameters are plotted against Rasch parameter estimates. (The latter were obtained using the Multilog software by Thissen et al. 2003.)

The ordering of the items is reflected in both sets of category quantifications. Furthermore, the person scores give a similar ordering compared with the sum score or item-response-theory estimates. Because MCA assigns different scores to different score patterns with the same sum score, the person scores and sum scores are close but not the same. 


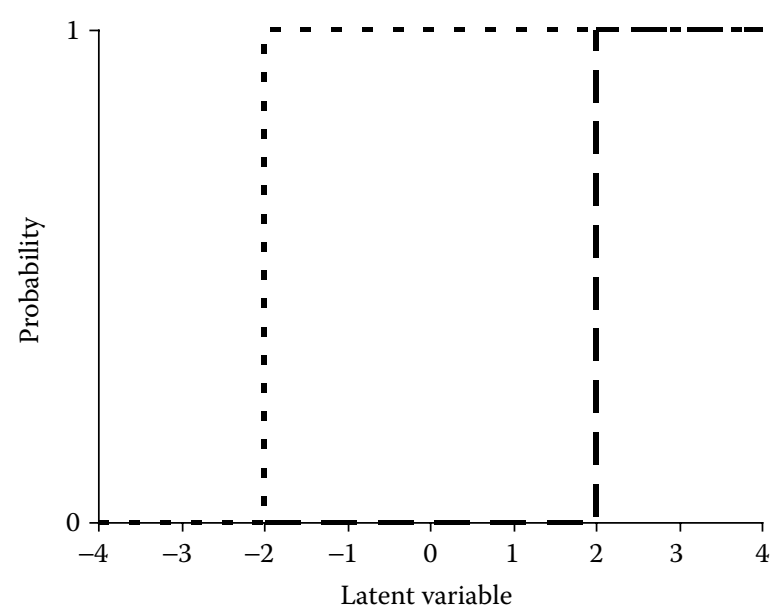

Figure 9.6 Two item-step functions of a PGS.

\subsection{The polytomous Guttman scale}

The extension of the DGS to more than two categories is the polytomous Guttman scale (PGS). An item is now characterized by two or more item-step functions, as shown in Figure 9.6.

The number of possible score patterns for a PGS is $Q(J-1)+1$. With $J>2$, various combinations of score patterns can form a PGS, so the PGS is not unique. Three interesting PGSs can be identified in the case $Q=2$ and $J=3$ :

$$
\mathbf{M}_{1}=\left[\begin{array}{ll}
1 & 1 \\
2 & 1 \\
2 & 2 \\
3 & 2 \\
3 & 3
\end{array}\right]
$$$$
\mathbf{M}_{2}=\left[\begin{array}{ll}
1 & 1 \\
2 & 1 \\
3 & 1 \\
3 & 2 \\
3 & 3
\end{array}\right]
$$$$
\mathbf{M}_{3}=\left[\begin{array}{ll}
1 & 1 \\
2 & 1 \\
2 & 2 \\
2 & 3 \\
3 & 3
\end{array}\right]
$$

The score patterns of $\mathbf{M}_{1}$ are such that the easier item steps of both items are taken first. Hence, there is an ordering of items within item steps (IWIS). A property of an IWIS PGS is that it has a maximum number of entries of the middle categories and a minimum number of entries of the two outer categories. 
The score patterns of $\mathbf{M}_{2}$ are such that both item steps of the first item are taken first, and then the item steps of the second item are taken. Thus, there is a complete ordering by items: all item steps of the first item are easier to take than the item steps of the second item. Hence, there is an ordering of item steps within items (ISWI). A property of an ISWI PGS is that it has a maximum number of entries of the two outer categories and a minimum number of entries of the middle categories.

The score patterns of $\mathbf{M}_{3}$ are such that all item steps of one item lie between the item steps of the other item, that is, item within item (IWI).

Similar to the different forms of the Rasch model, the various forms of the PGS also have consequences for the application of MCA. Only for the IWIS PGS can the rows and columns of the indicator coding be reshuffled such that there are consecutive ones for both rows and columns. The consecutive-ones property for rows indicates that, in a binary pattern, there is only one row of ones and either one or two rows of zeros. A similar property can be formulated for the columns.

$$
\mathbf{M}_{1}=\left[\begin{array}{ll}
1 & 1 \\
2 & 1 \\
2 & 2 \\
3 & 2 \\
3 & 3
\end{array}\right] \Rightarrow \mathbf{Z}_{1}=\left[\begin{array}{llllll}
1 & 0 & 0 & 1 & 0 & 0 \\
0 & 1 & 0 & 1 & 0 & 0 \\
0 & 1 & 0 & 0 & 1 & 0 \\
0 & 0 & 1 & 0 & 1 & 0 \\
0 & 0 & 1 & 0 & 0 & 1
\end{array}\right] \rightarrow\left[\begin{array}{llllll}
1 & 1 & 0 & 0 & 0 & 0 \\
0 & 1 & 1 & 0 & 0 & 0 \\
0 & 0 & 1 & 1 & 0 & 0 \\
0 & 0 & 0 & 1 & 1 & 0 \\
0 & 0 & 0 & 0 & 1 & 1
\end{array}\right]
$$

Hence, MCA can order both categories and persons of an IWIS PGS (see Hill 1974; Heiser 1981), which results in a parallelogram pattern, which in turn gives an arch in the two-dimensional solution. In Figure 9.7, the two-dimensional MCA person scores of an IWIS, ISWI, and IWI PGS, all complete and uniform, are plotted. Each PGS consists of scores of 13 persons on three items with five categories.

The ordering of the persons of an IWIS PGS is clearly illustrated with the arch in Figure 9.7. However, for the ISWI PGS, MCA cannot distinguish between several score patterns: in Figure 9.7 some person scores in the two-dimensional solution for the ISWI PGS are the same, although the original score patterns are different. Although it is not shown here, it is interesting to note that several item categories of the ISWI PGS also could not be distinguished. Combinations of these item categories and the corresponding persons obtain the same scores on different dimensions. In Figure 9.7, the original 13 ISWI score patterns are clustered in five groups.

It is easily shown that there exist no permutations for rows and columns such that the indicator matrix of the IWI PGS will have a 

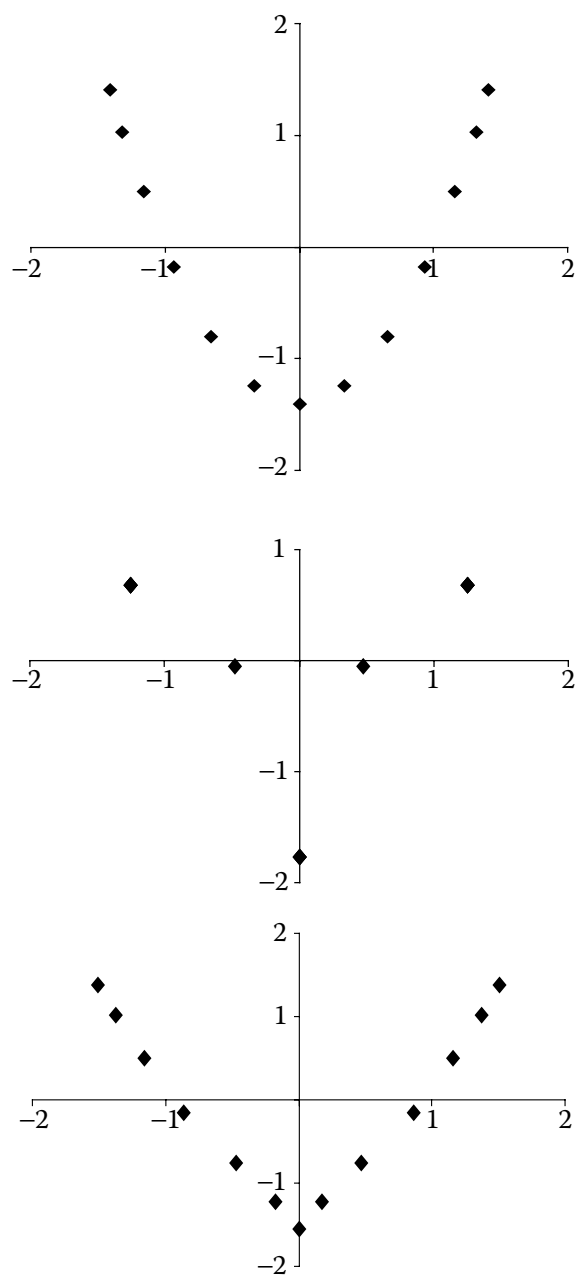

Figure 9.7 Plots of 13 MCA person scores in two dimensions for IWIS, ISWI, and IWI PGSs; $Q=3, J=5$.

parallelogram pattern, although it is possible to obtain a pattern that is very close to a parallelogram. This may account for the two-dimensional visualization presented in Figure 9.7 of the IWI PGS. The IWI arch in Figure 9.7 is pointed, but it does reflect the correct ordering of the persons.

To obtain the same strong results for the PGS that hold for the DGS discussed in Section 9.2, one should analyze the item steps and 
not the original data matrix. It is easily shown that any PGS can be made into a DGS by recoding the data matrix of a PGS into item steps. (The authors are not aware of any work in the literature where this property is explicitly stated.)

$$
\mathbf{M}_{2}=\left[\begin{array}{ll}
1 & 1 \\
2 & 1 \\
3 & 1 \\
3 & 2 \\
3 & 3
\end{array}\right] \Rightarrow \mathbf{M}_{2}^{\mathrm{IS}}=\left[\begin{array}{llll}
1 & 1 & 1 & 1 \\
2 & 1 & 1 & 1 \\
2 & 2 & 1 & 1 \\
2 & 2 & 2 & 1 \\
2 & 2 & 2 & 2
\end{array}\right] \Rightarrow \mathbf{Z}_{2}^{\mathrm{IS}}=\left[\begin{array}{llllllll}
1 & 1 & 1 & 1 & 0 & 0 & 0 & 0 \\
0 & 1 & 1 & 1 & 1 & 0 & 0 & 0 \\
0 & 0 & 1 & 1 & 1 & 1 & 0 & 0 \\
0 & 0 & 0 & 1 & 1 & 1 & 1 & 0 \\
0 & 0 & 0 & 0 & 1 & 1 & 1 & 1
\end{array}\right]
$$

When one analyzes the item-step data matrix, the individual item steps are analyzed as if they were dichotomous items, and all results of the dichotomous case apply.

\subsection{The graded response model}

A possible generalization of both the Rasch model to more than two categories, as well as the PGS to the probabilistic case, is the graded response model (GRM) proposed by Samejima (1969). An item with three response categories is characterized by two item-step functions (see Figure 9.8).

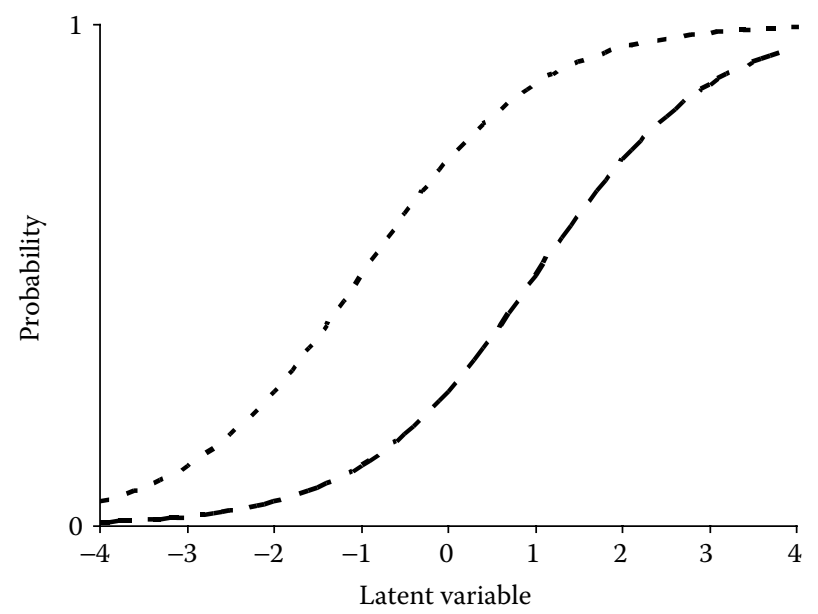

Figure 9.8 Two item-step functions of the GRM. 


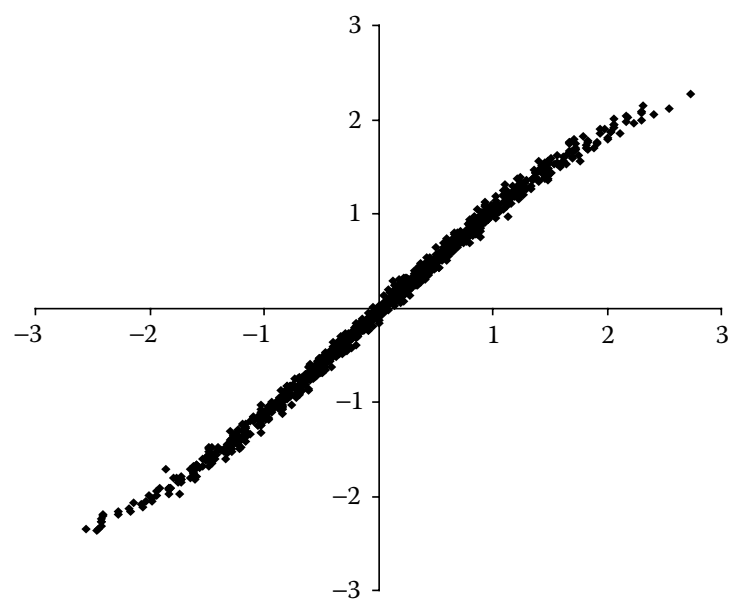

Figure 9.9 Plot of MCA person scores (vertical) vs. item-response-theory estimates (horizontal) for GRM.

Some results from Section 9.3 on the Rasch model also apply to the GRM. Depending on the steepness of the slopes, results very similar to those depicted in Figure 9.4 can be obtained for the GRM. Also, the category quantifications corresponding to the two outermost categories can be shown to be ordered under the same conditions as derived by Schriever (1985) for the Rasch model. An interesting graph shows the item-response-theory person estimates plotted against the MCA person scores of the first dimension (see Figure 9.9). This figure illustrates that the MCA person score is a reasonable approximation of the latent variable (see McDonald 1983; Cheung and Mooi 1994).

\subsection{Unimodal models}

It was Coombs (1964) who popularized the unidimensional unfolding technique and his method of parallelogram analysis. For the dichotomous Coombs scale (DCS), it holds that each item function is of unimodal shape instead of a monotonic one (see Figure 9.10).

With $Q$ items, the complete DCS has $2 Q+1$ score patterns. Its extension to more than two categories, the polytomous Coombs scale (PCS), will have $2 Q(J-1)+1$ possible score patterns in the complete case, i.e., the highest category has one category function, whereas the lower categories, except for the lowest, have category functions on both sides of the highest. 


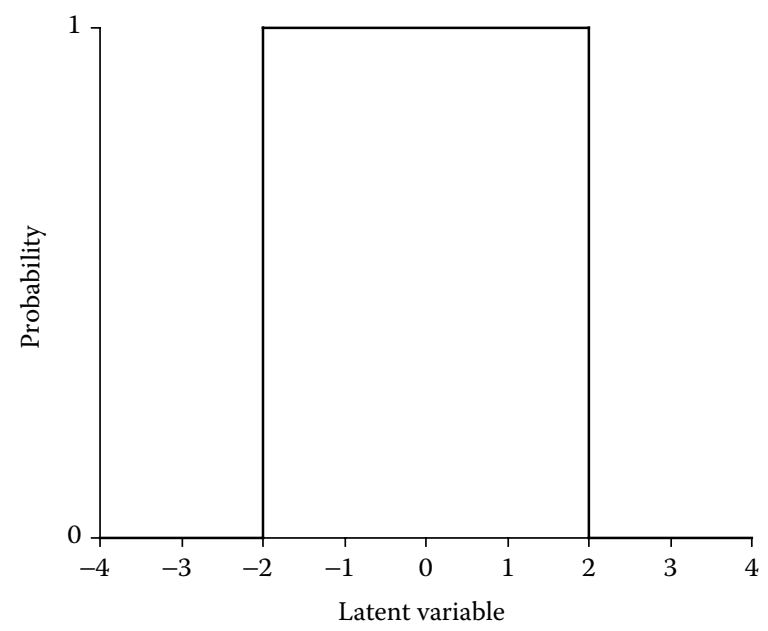

Figure 9.10 Item function of the DCS.

The list of literature on scaling the DCS or PCS with MCA is very short, or perhaps nonexistent. Heiser (1981: 120) demonstrated for a PCS that MCA does not find the intended regularity: neither the order of persons, nor the order of the items, nor the order of the categories within items are recovered in any simple way.

Where the DGS has one item step, which is also the item function, the item function of the DCS consists of two item steps, one left and one right. Some results have been obtained for the restricted DCS, i.e., the DCS where the left and right item steps have the same ordering across items. The restricted DCS can be analyzed by not assigning weights to both categories, but just to the category of interest, that is, simple CA of the $(0,1)$-table. Then it holds that the CA solution of the restricted DCS provides an ordering for both items and persons (see Hill 1974; Heiser 1981).

$$
\mathbf{M}=\left[\begin{array}{lll}
1 & 1 & 1 \\
2 & 1 & 1 \\
2 & 2 & 1 \\
1 & 2 & 1 \\
1 & 2 & 2 \\
1 & 1 & 2 \\
1 & 1 & 1
\end{array}\right] \Rightarrow\left[\begin{array}{lll}
0 & 0 & 0 \\
1 & 0 & 0 \\
1 & 1 & 0 \\
0 & 1 & 0 \\
0 & 1 & 1 \\
0 & 0 & 1 \\
0 & 0 & 0
\end{array}\right] \rightarrow \mathbf{M}^{0}=\left[\begin{array}{lll}
1 & 0 & 0 \\
1 & 1 & 0 \\
0 & 1 & 0 \\
0 & 1 & 1 \\
0 & 0 & 1
\end{array}\right]
$$


A possible generalization of the above coding to the PCS, called "conjoint coding" (as opposed to "disjoint" or "indicator coding" used in ordinary MCA), has been proposed by Heiser (1981). Alternatively, to successfully apply MCA to the DCS or PCS, one should make the distinction between the lower categories that lie on the left and on the right of the highest category. This is possible for both the DCS and PCS, because both models have deterministic data structures. The following is an example for a PCS.

$$
\mathbf{M}=\left[\begin{array}{lll}
1 & 1 & 1 \\
2 & 1 & 1 \\
2 & 2 & 1 \\
2 & 2 & 2 \\
3 & 2 & 2 \\
3 & 3 & 2 \\
3 & 3 & 3 \\
2 & 3 & 3 \\
2 & 2 & 3 \\
2 & 2 & 2 \\
1 & 2 & 2 \\
1 & 1 & 2 \\
1 & 1 & 1
\end{array}\right] \Rightarrow \mathbf{M}^{\mathrm{D}}=\left[\begin{array}{lll}
1 & 1 & 1 \\
2 & 1 & 1 \\
2 & 2 & 1 \\
2 & 2 & 2 \\
3 & 2 & 2 \\
3 & 3 & 2 \\
3 & 3 & 3 \\
4 & 3 & 3 \\
4 & 4 & 3 \\
4 & 4 & 4 \\
5 & 4 & 4 \\
5 & 5 & 4 \\
5 & 5 & 5
\end{array}\right]
$$

This idea is discussed for applications in the field of item-response theory by several authors (Verhelst and Verstralen 1993; van Schuur 1993c; Andrich 1996; Roberts and Laughlin 1996).

\subsection{Conclusion}

As shown in the previous sections of this chapter, the application of MCA to monotonic unidimensional models has a vast potential for producing interesting results. The application of MCA to unimodal models is somewhat less fruitful although some results could be obtained for the deterministic Coombs scales. The idea that one needs to consider what coding is appropriate for the multivariate categorical data at hand, before applying MCA, is probably the most important point of this chapter. A choice must be made between coding items or item steps, or between disjoint or conjoint coding of the data. But given 
this choice, MCA optimizes a general-purpose criterion, not a modelspecific one.

In Section 9.6, a coding scheme was discussed that made a distinction between the lower categories that lie on the left and on the right of the highest category of a PCS. What the reader may not have noted is that, by making this distinction, the PCS with $J$ categories becomes a PGS with $2(J-1)+1$ categories. Furthermore, all results from Section 9.4 on the PGS now also apply to the PCS. Even the DCS can be considered a PGS after recoding the data. Note that the item steps coding from Section 9.4 can be applied as well, in which case all properties from Section 9.2 also apply to the DCS and PCS. Thus, after the appropriate codings, the PGS, DCS, and PCS can be analyzed as if they were DGSs. A similar result holds for the probabilistic GRM. If one applies the item-steps coding to the GRM data, applying MCA becomes the same as analyzing dichotomous item steps with the Rasch model.

From the figures in this chapter, it is clear that the common conviction-that applying MCA to data corresponding to a unidimensional model always results in a horseshoe or an arch-is not true. This finding even holds for relatively less complex unidimensional models such as the PGS or the Rasch model. Even though an arch is not necessarily obtained with probabilistic models, Figure 9.5 and Figure 9.9 demonstrate that MCA contains relevant information on the person and sometimes the location parameters of a monotonic model in its first solution. For each of the deterministic models, a vast number of possible probabilistic generalizations exist. This chapter has been limited to only a few of them. What has not been shown here, but what is interesting to note, is that models that are more complex than the basic Rasch model, for example, the two-dimensional plot (or higherdimensional plots), are very unclear and hard to interpret. However, even if the two-dimensional plot looks like random data, the first MCA dimension contains relevant information on at least the person parameters of monotonic unidimensional models (see Figure 9.5 and Figure 9.9). The person score seems a reasonable approximation of the latent variable for a wide variety of models. This latter property should be interpreted by the MCA community as a critical note against the sometimes blind use of two-dimensional plots. 